\title{
Community Awareness - A Key to the Early Detection of Head and Neck Cancer
}

Ajay Manickam, ${ }^{1}$ Jayanta Saha, ${ }^{1}$ Debangshu Ghosh, ${ }^{1}$ Shaswati Sengupta, ${ }^{1}$ Debabrata Biswas, ${ }^{1}$ Sumit Kumar Basu, ${ }^{1}$ Satish Kumar, ${ }^{2}$ Souradeep Ray ${ }^{1}$

Introduction

ABSTRACT

Mortality and morbidity associated with head and neck cancers have decreased to a great extent in many developed countries of the world due to early diagnosis and treatment with advances in surgical techniques and better availability of radiotherapist and oncologists. But the situation in developing countries like India is quite different.

Aims and Objectives

This study was conducted amongst the patients attending the Otolaryngology department of a teaching hospital in Kolkata to find the incidence of various types and sites of head and neck cancer, to assess time delay from the date of onset of symptom to the final disease confirmation in relation to patients' demographic profile, to assess time delay in histopathological diagnosis after reaching a tertiary care setup and also to correlate tobacco consumption and alcohol intake as risk factors for head and neck cancer and to note the reasons for late presentation, as described by the patient.

Materials And Methods

The descriptive study was conducted at a tertiary level teaching hospital, in the Department of Otorhinolaryngology for a period from August 2013 to August 2015 with a study population of 133.

Observations

An average time lag from the onset of symptom to final diagnosis as malignancy was found to be 6 months to one year in nearly $72 \%$ of cases. Most of the patients were uneducated males of more than 50 years of age, hailing from rural areas. Cancer larynx was found to be the commonest of all head and neck cancers (31.6\%). More than $65 \%$ of the patients were addicted to tobacco chewing or smoking or consumption of alcohol.

Discussion

Poverty, lack of education, poor communication, lack of health care infrastructure in rural areas, community awareness about various risk factors, lack of effective health policy to achieve early diagnosis of head neck cancer were common factors related to delay in diagnosis.

Conclusion

Tobacco use and alcohol intake are the modifiable risk factors of head and neck cancer. Educating the field workers about the danger signs of head and neck cancer may promote early referral of the suspected cases to tertiary health care setup for early detection of cancer.

$\underline{\text { Kevwords }}$

Early Detection of Cancer; Head and Neck Neoplasms; Developing Countries; Risk Factors

$\mathrm{M}$ ortality and morbidity associated with head and neck cancers have decreased to a great extent in many developed countries of the world. Early medical advice seeking behaviour, advanced surgical techniques and availability of radiotherapist and oncologists are much better in developed world. But in developing countries like India it's not so.

Mortality due to cancer in India is around 6,82,830.
Among them mortality due to head and neck cancer is around 1,08,537 in which 82,290 are males and 26,247

1 - Department of ENT, R G Kar Medical College, Kolkata 2 - Department of Community Medicine, T N MGR Medical University

Corresponding author:

Dr Ajay Manickam

email: ajaymanickam87@gmail.com 
are females. ${ }^{1}$ Although government of India has offered so much of cost effective medical services to the people, there is no screening programme proposed for the sake of head and neck cancer. ${ }^{2}$

Hence health education and proper medical healthcare seeking behaviour of the public is very important in order to decrease the mortality associated with head and neck malignancy. In India head and neck cancers are found to be more related in lower socioeconomic strata. As patients presenting in tertiary care government hospitals mostly belong to the low socio-economic group and also advanced stage disease are commonly seen in rural population, ${ }^{2}$ their awareness about cancer has been found to be low. When head and neck cancers are diagnosed at a later stage, survival rate is poor.

In India nearly 1,08,537 people die each year from head and neck cancer. ${ }^{1}$ Most of the patients seek medical advice at a very late stage in a tertiary care setup. Head and Neck cancers are the most common cause of deaths due to malignancy in India. Community awareness about cancer and its treatment options is generally very poor. This study deals with the delay in diagnosis and community awareness of head and neck cancer.

\section{Subsites in the head and neck region}

In Head and neck cancer the TNM (Tumour, Nodes, Metastases) classification applies to carcinomas of the following sites: lip, oral cavity, pharynx (oropharynx, nasopharynx, hypopharynx), larynx, maxillary sinus, nasal cavity, ethmoid sinus, salivary glands and thyroid gland. ${ }^{3}$ Each site is described with rules for classification, anatomical sites and sub sites (where appropriate), the TNM classification, grading for histopathology, staging and summary. The TNM groups then are reassigned to four groups - stage groups (stages one - four). This staging helps the clinician to aid in the planning and evaluation of results and is one of the indicators of prognosis. ${ }^{4}$

\section{Aims and Objectives}

1. To find the various types and sites of head and neck cancer

2. To assess time delay from the date of onset of symptom to the final disease confirmation in relation to patents demographic profile

3. To assess time delay in histopathological diagnosis after reaching a tertiary care setup

4. To correlate tobacco use and alcohol consumption as risk factors for head and neck cancer

5. Various reasons as described by the patient for late presentation

\section{Materials and Methods}

The study was conducted at a tertiary level teaching hospital in Kolkata in the Department of Otorhinolaryngology from August 2013 to August 2015. This descriptive study was undertaken after getting clearance from the Institutional Ethics Committee and informed consent was obtained from all the participants. The patients meeting the clinical criterion with features of head and neck malignancy were included in the study.

A thorough history was taken as per preformed questionnaire along with detailed clinical examination, endoscopic and radiological evaluation were done. Importance was given to four important dates as follows, (1) date of onset of first symptom, (2) date of seeking first medical advice. (3) date of visiting tertiary care setup, (4) date of final histopathological confirmation as cancer. This health care professional contacted could either be a homeopathic doctor, Ayurvedic doctor, quack, allopathic general practitioner or a specialist. Reasons for the late presentations were also recorded as said by the patient. The patient's socio economic and education status were collected according to the Modified Kuppusamy classification. The site of lesion, histopathological type of the cancer was also individually recorded and the tabulation of results were done.

Patients with clinically suggestive malignancy, who gave consent for the study, were included in the study group. Elderly debilitated patients, who were lost to follow up and those who did not give consent for the study, were excluded. A total of 133 patients were enrolled in this study. Demographic analysis and analysis of variance (ANOVA) charts were made in SPSS software and the results were tabulated. 


\section{Observations}

Among the study population of 133,85 patients were male $(63.9 \%)$ and 48 were female (36.1\%) (Fig. 1 ). More than $70 \%$ of the study population were of age greater than 50 years (Fig. 2). Majority of the patients were elderly males. Nearly $82.3 \%$ of the study population (Fig. 3) was from peripheries of Kolkata (greater than $50 \mathrm{~km}$ from Kolkata).

Majority of the study population also belonged to the low socio-economic status (Fig. 4) as they were either daily labourers or farmers $(62.5 \%)$. More than $65 \%$

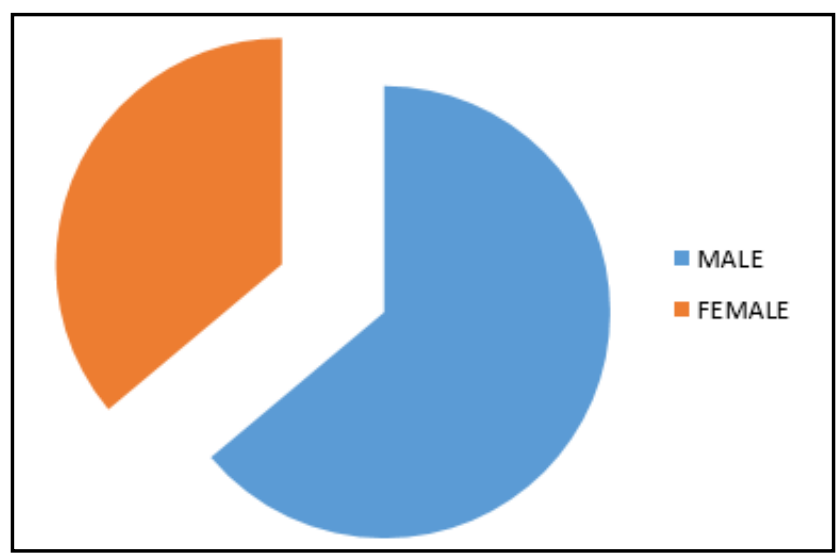

Fig. 1 Distribution according to sex

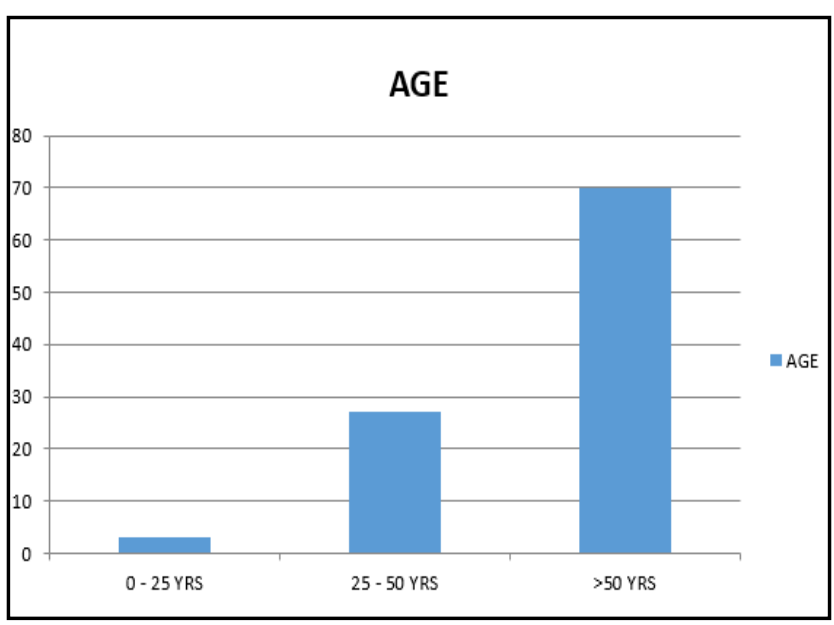

Fig. 2 Distribution according to age

of the population was addicted to tobacco chewing or

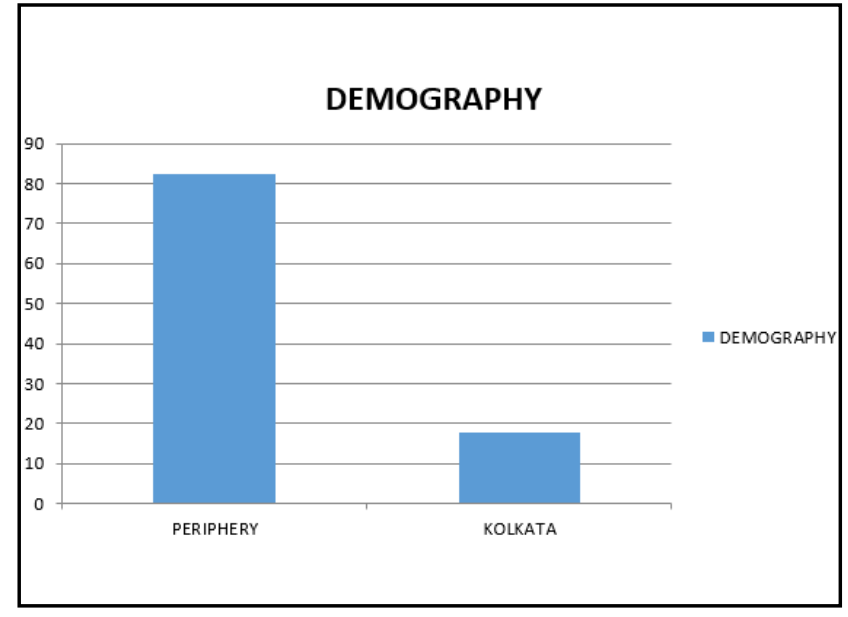

Fig. 3 Demography

smoking or consumption of alcohol. Nearly $53.3 \%$ of the study population was having habits of both smoking and alcohol consumption and nearly $9.8 \%$ of the population were having habits of both tobacco chewing and smoking. According to modified Kuppusamy scale education status were tabulated. It was found that majority of the population were uneducated $(78.9 \%)$.

Cancer larynx was the most common of all head and neck cancers $(31.6 \%)$ and temporal bone cancer $(0.8 \%)$ was the least common of all (Fig. 5). Among the cancer subtypes, squamous cell carcinoma (Fig. 6) was found to be the most common $(82 \%)$.

\section{SOCIO ECONOMIC STATUS}

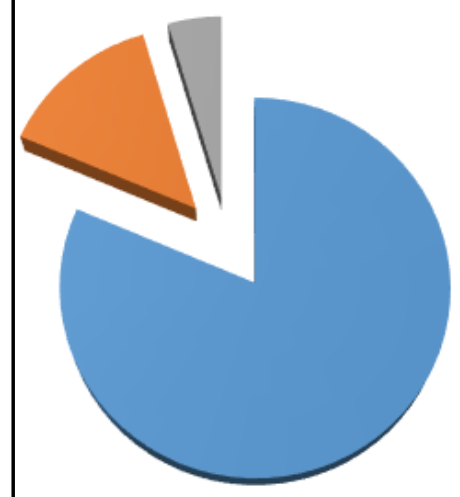

I POOR

MIDDLE CLASS

UPPER CLASS

Fig. 4 Socioeconomic status 


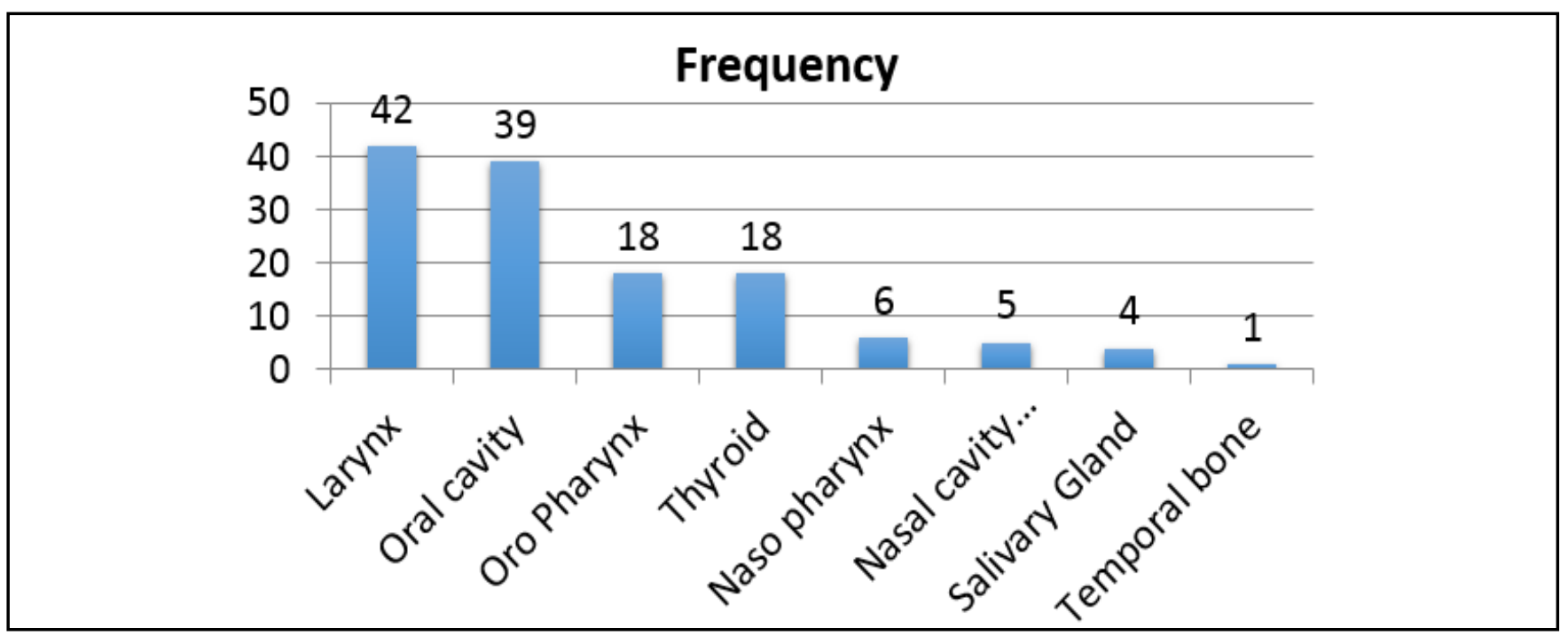

Fig. 5 Sites of Cancer

On calculating the time delay at various intervals, nearly $65.4 \%$ population sought medical advice for the first time in less than 6 months of time (Fig. 7) (visiting either a quack, homeopathy doctor, general practitioner or a specialist). After getting this medical advice, nearly $41.4 \%$ population took a time period of about 6 months and $30.7 \%$ population took a time period of greater than one year to visit a tertiary care hospital.

After reaching a tertiary setup to get finally diagnosed as malignancy, $68 \%$ of study population needed another 2 months and $4.2 \%$ greater than two months. So an average time lag from the onset of symptom to final

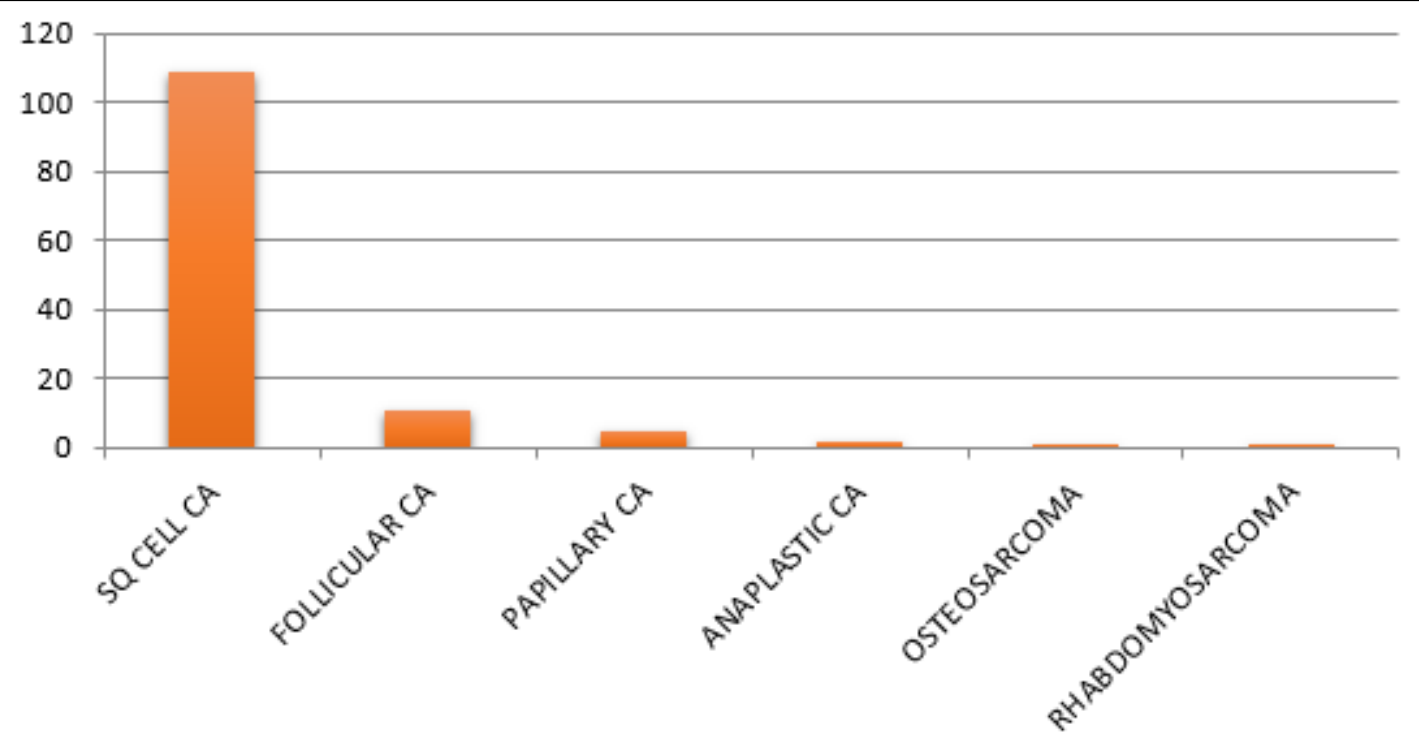

Fig. 6 Histopathological types of cancer 
diagnosis as malignancy was found to be at least 6 months to one year in nearly $72 \%$ of cases (Table I). Nasopharyngeal cancers were the earliest and thyroid cancers were the last to be diagnosed (Table II).

The relation of this time lag to age, sex, religion, type of cancer, site of cancer, socio-economic status, were all found to be of statistically insignificant value. But the relationship of medical advice seeking behavior (Table III) with education status $(\mathrm{p}=0.0441)$, relationship of time lag with people living near to tertiary care setup $(\mathrm{p}=0.0397)$, were found to be statistically significant. The relationship of tobacco use and alcohol intake with cancer was also found to be statistically significant $(\mathrm{p}=0.045)$.

\section{Discussion}

Clinicians managing head and neck cancers have a responsibility to assess the outcome and prognosis. In this perspective, collection of data with a thorough preformed questionnaire is very essential. Head and neck cancer includes carcinomas of the following sites: lip, oral cavity, pharynx (oropharynx, nasopharynx, hypopharynx), larynx, maxillary sinus, nasal cavity, ethmoid sinus, salivary glands and thyroid gland.3 Staging the disease helps the clinician to aid in the planning and evaluation of results and is one of the indicators of prognosis. ${ }^{4}$

Head and neck cancers, according to WHO data, are

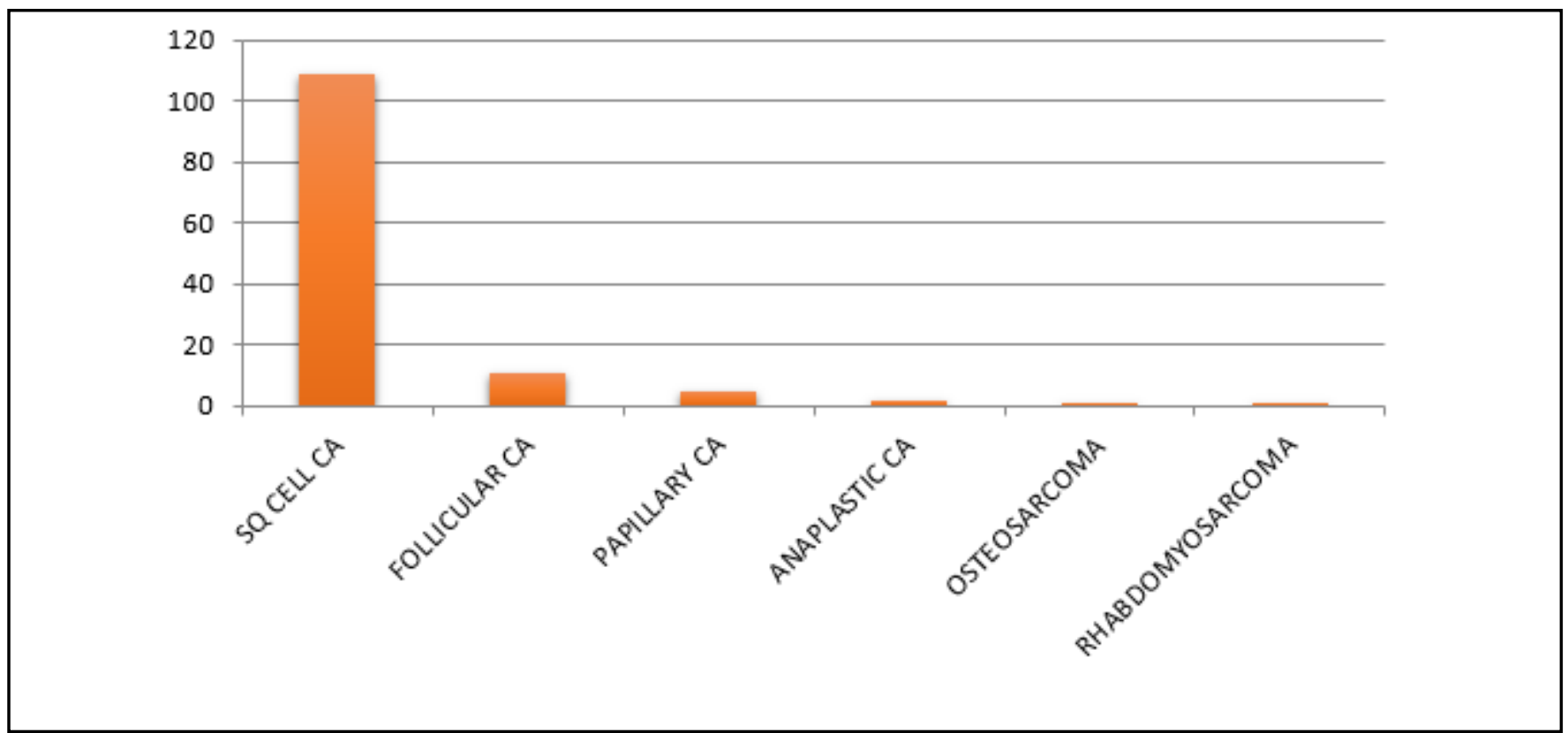

Fig. 7 First point of Healthcare Contact

Apart from the analysis, the reason for late presentation was asked as a direct question to all the study population. They gave multiple answers. All the answers were tabulated. $64.5 \%$ of the study population said poor socio economic status to be the commonest cause, followed by $58 \%$ people who replied medical services were far away from their place; $36 \%$ were also not aware of the gravity of the lesion. seen more commonly in males compared to females. ${ }^{5}$ In our study also it was found that majority of the study population were male $(63.9 \%)$.

In India, most of the people seeking government tertiary care setup belong to very low socio-economic status. ${ }^{6}$ Study group contained about $62.5 \%$ population belonging to very poor socio-economic condition.

According to WHO, cancer of the mouth and oral 


\section{Date of first symptom to first medical advice}
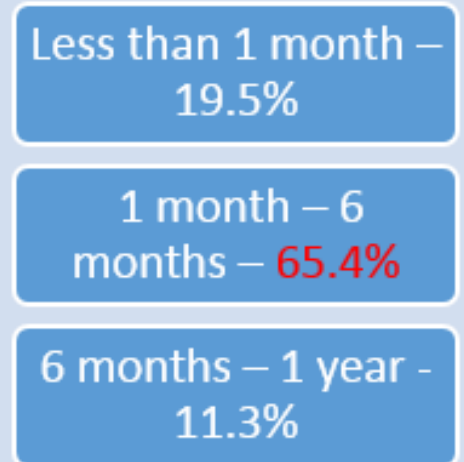

Greater than 1 year $-3.8 \%$

\section{First medical advice to tertiary care hospital}

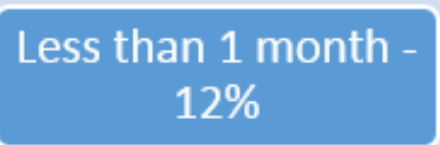

1 month -6

months $-41.4 \%$

6 months - 1 year $15.8 \%$

Greater than 1 year $-30.7 \%$

\section{After reaching tertiary care setup and final diagnostic confirmation}

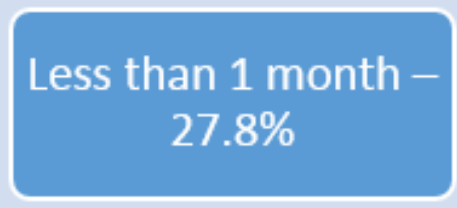

1 month -2

months $-68 \%$

Greater than 2

months $-4.2 \%$

Table I Time lag in reaching a tertiary care centre after the onset of symptoms and the final diagnostic confirmation

cavity are the commonest malignancy in males in India but in females cervical and breast cancers are most common. $^{7}$

In India, two-thirds of the population live in rural areas. $^{8}$ Poor communication, poverty, lack of healthcare infrastructure in rural areas, community awareness about various risk factors associated with tobacco use, lack of effective health policy to achieve early diagnosis of head neck cancer are common factors related to delay in diagnosis. Socio-economic and demography play important roles in determining the early medical advice seeking behavior. In this study also majority of the study population belong to places distant from Kolkata (more than $50 \mathrm{~km}$ away).

We observed that medical advice seeking behavior of the patient is unsatisfactory. People of rural areas prefer visiting a quack or a homeopath rather than visiting a general practitioner. Majority of the population visits a homeopath, quack or an Ayurveda doctor (69.8\%), rather than visiting a $\mathrm{PHC}$ or a specialty centre.

It is clearly evident (Table I) that there is a significant delay in the final diagnosis of malignancy from the day of onset of first symptom. Average of 6 months to one year time delay is observed in nearly $70 \%$ of the study population.

It is also clear that nasopharyngeal cancer were the earliest to be diagnosed and thyroid cancer was the one with late presentation of about 20 years (Table II).

The commonest reasons, as stated by the patients for late presentation, were socio-economic factors (64\%) and demography(58\%). $36 \%$ of the patients were not aware that it could be a malignancy.

WHO defines a healthy nation according to various morbidity indicators, mortality indicators, health 
determinant factors such as tobacco use, alcohol use etc. In India tobacco is not banned. Banning tobacco use can prevent $22 \%$ of death due to cancer. If this same scenario persists, $70 \%$ increase in head and neck cancer patients can be expected in the next two decades. ${ }^{9}$

In India there are no cancer screening programmme for head and neck cancer detection. Tobacco use is not completely banned in many of the states in India. In north eastern India nearly $50 \%$ of cancer is due to head and neck malignancy. ${ }^{10}$ Death due to head and neck cancer is one among the top 10 leading causes of death in India.

This time delay in diagnosing if properly assessed and if community awareness is increased, mortality due to head and neck cancer can be very much reduced. In recent times in India Revised National Tuberculosis Programme, Universal Immunization Programme, National AIDS Control Programme have all met with tremendous success because of field workers. Community awareness about immunization has very much improved.

Hence various suggestions from this study are, danger signs of head and neck cancer must be formulated and cancer screening programme for head and neck malignancy in the high risk group has to be initiated by the government. Studies have proved that effective screening can considerably increase the early detection of cancer.

Field workers should be initiated like various other national programmes, and important danger signs of head and neck cancer such as hoarseness of voice, nonhealing ulcer in the oral cavity, swelling in the neck, nasal cavity, all has to be noted up properly by the field workers and they have to be instructed regarding the condition and has to be referred immediately to the tertiary care centre, so that early detection and diagnosis can be established. Cost effective screening which can detect cancers at an early, treatable and less costly stage, is an important part of delivering affordable cancer care in India. ${ }^{2}$

\section{Conclusion}

Tobacco use and alcohol intake are the modifiable risk factors of head and neck cancer. Tobacco control can significantly decrease the incidence of head and

Table II : Types of cancer and their relationship to time taken for the diagnosis

\begin{tabular}{|c|c|c|}
\hline $\begin{array}{c}\text { SITE OF } \\
\text { CANCER }\end{array}$ & $\begin{array}{c}\text { MINIMUM } \\
\text { DAYS }\end{array}$ & $\begin{array}{c}\text { MAXIMUM } \\
\text { DAYS }\end{array}$ \\
\hline Nasopharynx & 17 & 94 \\
\hline Salivary gland & 30 & 212 \\
\hline Larynx & 82 & 1264 \\
\hline Oral cavity & 91 & 1157 \\
\hline Oropharynx & 122 & 1673 \\
\hline Sinonasal & 441 & 3407 \\
\hline Thyroid & 479 & 6774 \\
\hline
\end{tabular}

neck malignancy. Living in rural areas and poor socio-economic conditions are important hindrances in the early detection of cancer. By implementing a community awareness protocol and an effective screening programme for head and neck cancer, it can be easily diagnosed early. Knowledge about danger signs of head and neck cancer among the field workers can ensure early referral to tertiary setup and hence, early diagnosis.

Table III : Analysis of variance statistically significant variables

\begin{tabular}{|c|c|}
\hline $\begin{array}{c}\text { ANALYSIS OF VARIANCE } \\
\text { STATISTICALLY SIGNIFICANT } \\
\text { VARIABLES }\end{array}$ & P VALUE \\
\hline $\begin{array}{l}\text { Time lag with people living near to } \\
\text { a hospital }\end{array}$ & 0.0397 \\
\hline $\begin{array}{l}\text { Medical advice seeking behaviour } \\
\text { to education status }\end{array}$ & 0.0441 \\
\hline $\begin{array}{l}\text { Relationship of tobacco use and } \\
\text { alcohol intake with head neck cancer }\end{array}$ & 0.045 \\
\hline
\end{tabular}




\section{References}

1. http://globocan.iarc.fr/Pages/fact_sheets_population.aspx. Last accessed on 19/02/2016

2. Nelson R. Cancer Incidence Increasing, Mortality High. Medscape - India May 06, 2014. www.medscape.com/ viewartcle/824697

3. Sobin LH, Wittekind CH. TNM Classification of malignant tumours, UICC, 6th edn. New York; Wiley-Liss, 2002. A summary and cornerstone for accurate staging.

4. Greene F, Page DL, Flemming ID, Fritz AG, Balch CM, Haller DG(eds). The AJCC cancer staging manual, 6th edn. New York; Springer, 2002. A summary and cornerstone for accurate staging.
5. http;//publications.iarc.fr/ International agency for research on cancer

6. Alvarez-Uria G, Middle M, Naik PK. Patients seeking medical advice in a Rural Hospital of India. J Public Health Res. 2012; 14:79-82. Doi.10.4081/jphr.2012.e14. ecollection 2012.

7. http;//www.who.int/topics/cancer/en

8. www.tradingeconomics.com/india/rural-population-percentof-total-population-wb-data.html. Last accessed on 19/02/2016

9. globocan.iarc.fr/Pages/fact sheets_cancers.aspx-international agency for research on cancer. GLOBOCAN 2012; estimated cancer incidence, Mortality and prevalence worldwide in 2012. Last accessed on 19/02/2016

10. Trivedi NP, Kekatpure VD, Trivedi NN, Kuriakose MA. Head and Neck cancer in India. Need to formulate uniform national treatment guideline? Indian Journal of Cancer 2012; 49:6-10. doi: 10.4103/0019-509X.98907 